\title{
Calnexin, an ER-induced protein, is a prognostic marker and potential therapeutic target in colorectal cancer
}

\author{
Deborah Ryan ${ }^{1,2}$, Steven Carberry ${ }^{1}$, Áine C. Murphy ${ }^{1}$, Andreas U. Lindner', Joanna Fay ${ }^{3}$, Suzanne Hector ${ }^{1}$, \\ Niamh McCawley ${ }^{1,2}$, Orna Bacon ${ }^{1,2}$, Caoimhin G. Concannon ${ }^{1,2}$, Elaine W. Kay ${ }^{3}$, Deborah A. McNamara² \\ and Jochen H. M. Prehn ${ }^{*}$
}

\begin{abstract}
Background: Colorectal cancer (CRC) is a leading cause of cancer mortality in the Western world and commonly treated with genotoxic chemotherapy. Stress in the endoplasmic reticulum (ER) was implicated to contribute to chemotherapeutic resistance. Hence, ER stress related protein may be of prognostic or therapeutic significance.

Methods: The expression levels of ER stress proteins calnexin, calreticulin, GRP78 and GRP94 were determined in $\mathrm{n}=23$ Stage II and III colon cancer fresh frozen tumour and matched normal tissue samples. Data were validated in a cohort of $n=11$ rectal cancer patients treated with radiochemotherapy in the neoadjuvant setting. The calnexin gene was silenced using siRNA in HCT116 cells.

Results: There were no increased levels of ER stress proteins in tumour compared to matched normal tissue samples in Stage II or III CRC. However, increased calnexin protein levels were predictive of poor clinical outcome in the patient cohort. Data were validated in the rectal cancer cohort treated in the neoadjuvant setting. Calnexin gene-silencing significantly reduced cell survival and increased cancer cell susceptibility to $5 \mathrm{FU}$ chemotherapy.
\end{abstract}

Conclusion: Increased tumour protein levels of calnexin may be of prognostic significance in CRC, and calnexin may represent a potential target for future therapies.

Keywords: Colorectal cancer, ER Stress, Calnexin, GRP78, GRP94, UPR (unfolded protein response)

\section{Background}

Colorectal cancer is one of the most common cancers among men and women in the Western world and the third leading cause of cancer-related mortality. Current treatment options for patients are dependent on disease stage at diagnosis but include surgery and adjuvant chemotherapy [1]. The most common chemotherapy regime currently used in the treatment of Stage II and III colorectal cancer is 5-fluorouracil (5FU), often in combination with oxaliplatin or irinotecan depending on a

\footnotetext{
*Correspondence: prehn@rcsi.ie

1 Department of Physiology and Medical Physics, Centre for Systems Medicine, Royal College of Surgeons in Ireland, 123 St Stephen's Green, Dublin 2, Ireland

Full list of author information is available at the end of the article
}

patients' clinical status [2, 3]. For patients with Stage II node negative disease, it is unclear if adjuvant therapy will be beneficial, as the majority of these patients will respond to surgery alone, with only a small subset of patients deriving benefit from adjuvant chemotherapy [4]. Adjuvant 5FU-based chemotherapy in stage III disease benefits $c$. $15-20 \%$ of patients, yet the majority of patients will relapse or develop distant metastases within 5 years following surgery [5]. It is therefore important that we improve our ability to identify patients who will benefit from adjuvant chemotherapies and to discover novel drug targets for those patients resistant to current chemotherapeutics.

The endoplasmic reticulum (ER) is an essential cellular organelle responsible for the synthesis; maturation and trafficking of a wide range of proteins and is a critical site 
for calcium homeostasis. Due to its central role in protein folding and quality control, the ability of the ER to adapt to adverse conditions is essential to the survival of a cell $[6,7]$. ER stress is triggered by the accumulation of unfolded or misfolded proteins in the ER and can result from an increased demand for protein synthesis, accumulation of mutant proteins, or from a pathophysiological interference with regular ER functions such as maintenance of $\mathrm{Ca}^{2+}$ homeostasis, hypoxia/ischemia or oxidative stress [8]. ER stress triggers the cell's unfolded protein response (UPR), which alters transcriptional and translational programmes within the cell. The goal of the UPR is to protect the cell from ER stress by decreasing the number of proteins translocated into the ER lumen, by increasing retrotranslocation and augmenting the protein folding capacity of the ER [7]. ER stress induces a number of different proteins such as chaperones from the heat shock protein family including GRP78 and GRP94; $\mathrm{Ca}^{2+}$ binding chaperone lectins such as calnexin and calreticulin; and substrate specific chaperones [7].

Somatic gene mutations as well as ischemic conditions may trigger ER stress and elicit the UPR in tumour cells. Rapid growth of tumours can lead to inadequate vascularisation resulting in decreased levels of oxygen, nutrients and glucose as well as altering intracellular calcium levels. In highly oncogenic tumours, protein overproduction or mutations found within tumour cells may cause mutant protein accumulation leading to ER stress $[6,9]$. ER stress proteins have been shown to be upregulated in a number of tumour types including breast, lung, liver and prostate tumours and it has been suggested that these altered levels of ER stress proteins are essential for the survival of cancer cells in the inhospitable tumour microenvironment $[7,10,11]$. Thus, in this study we examined whether the ER stress proteins GRP78, GRP94, calnexin and calreticulin are differentially expressed in colorectal tumours compared to matched normal tissue and determined if there was any association between the expression of ER stress proteins and disease stage or clinical outcome in our CRC patient cohort.

\section{Methods}

\section{Patients}

Patient tissue samples were collected and stored in the APOCOLON colorectal tissue biobank at Beaumont Hospital (Dublin, Ireland). Informed consent was received from all patients and ethical approval for use of the stored material was granted by Beaumont Hospital Ethics (Medical Research; 08/62, 11/09 and 16/20) Committee. Snap-frozen colorectal tumour and matched normal tissue from surgical resections of 23 Stage II $(\mathrm{n}=8)$ and III $(\mathrm{n}=15)$ colorectal cancer patients were prospectively collected. Fourteen of the stage III patients and one stage II patient received adjuvant chemotherapy with 10 receiving $5 \mathrm{FU}$ and leucovorin, 3 receiving $5 \mathrm{FU}$, leucovorin and oxaliplatin while 1 patient received $5 \mathrm{FU}$, leucovorin and irinotecan, and 1 patient received 5FU, leucovorin, irinotecan and bevacizumab. In 15 cases, patients had a good outcome which was defined as no mortality or disease recurrence within the 4-year followup period while 8 cases had a poor outcome, specifically, disease recurrence or death from disease within that timeframe.

Clinical follow-up was obtained for all patients and patient characteristics are summarised in Table 1.

\section{Validation cohort (neoadjuvant setting)}

Pre-treatment biopsies were also obtained from a separate cohort of rectal cancer patients by colonoscopy or rigid sigmoidoscopy and stored from the Departments of Surgery, and Pathology, Beaumont Hospital, Dublin, Ireland. Following treatment, surgical resections of these patients was obtained. Ethical approval was obtained by the Beaumont Hospital Ethics (Medical Research; 08/62, $11 / 09$ and 16/20) Committee, and informed consent was

Table 1 Details of the disease stage, chemotherapy treatment and disease outcome of the 23 patients within the study

\begin{tabular}{|c|c|c|c|}
\hline Patient & Stage & Received chemotherapy & Outcome \\
\hline 1 & $\|$ & None & Good \\
\hline 2 & $\|$ & None & Good \\
\hline 3 & $\|$ & None & Poor \\
\hline 4 & $\|$ & None & Good \\
\hline 5 & $\|$ & None & Good \\
\hline 6 & $\|$ & None & Poor \\
\hline 7 & $\|$ & None & Poor \\
\hline 8 & $\|$ & $5 F U+$ Leu & Good \\
\hline 9 & III & None & Good \\
\hline 10 & $\|$ & $\mathrm{Fol}+5 \mathrm{FU}+\mathrm{Oxal}+$ Irin & Poor \\
\hline 11 & III & $5 F U+$ Leu & Good \\
\hline 12 & III & $5 F U+L e u$ & Good \\
\hline 13 & III & $5 F U+$ Leu & Poor \\
\hline 14 & III & $5 F U+L e u$ & Good \\
\hline 15 & III & $\mathrm{Fol}+5 \mathrm{FU}+\mathrm{Oxal}$ & Good \\
\hline 16 & III & $\mathrm{Fol}+5 \mathrm{FU}+\mathrm{Oxal}$ & Poor \\
\hline 17 & III & $5 F U+L e u$ & Good \\
\hline 18 & III & $\mathrm{Fol}+5 \mathrm{FU}+\mathrm{Oxal}$ & Poor \\
\hline 19 & III & $5 F U+L e u$ & Good \\
\hline 20 & $\|$ & $5 F U+$ Leu & Good \\
\hline 21 & III & $5 \mathrm{FU}+$ Leu + Irin + bevacizumab & Poor \\
\hline 22 & III & $5 F U+$ Leu & Good \\
\hline 23 & III & $5 F U+$ Leu & Good \\
\hline
\end{tabular}

5FU 5-fluorouracil; Fol Fluorouracil; Irin irinotecan; Leu leucovorin; Oxal oxaliplatin; None no chemotherapy received 
obtained from all patients. Resections were evaluated to ensure consistent quality and tumour presence by an experienced pathologist. Clinical follow-up and response to neoadjuvant radio chemotherapy was obtained for all patients and patient characteristics are summarised in Table 2. After neoadjuvant chemo-radiotherapy, patients' response to therapy was determined using RCPath criteria. Good responders were identified as patients showing complete tumour progression (RCPathA). Poor responders were identified as patients showing partial or no marked tumour regression (RCPathB and C, respectively).

\section{Western blotting}

For clinical samples, tissue was lysed in ice-cold tissue lysis buffer [ $50 \mathrm{mmol} / \mathrm{L}$ HEPES (pH 7.5), $150 \mathrm{mmol} / \mathrm{L}$ $\mathrm{NaCl}, 5 \mathrm{mmol} / \mathrm{L} \mathrm{Na-EDTA}$ ] and protease inhibitor cocktail (Calbiochem, Hampshire UK) followed by mechanical homogenization on ice. Following centrifugation $(14,000 \times g$ for $10 \mathrm{~min})$, supernatant was collected and stored at $-80^{\circ} \mathrm{C}$ until further use. For the cell line experiments, cells were trypsinised and cell pellets collected for each experiment. Cell pellets were then lysed with SDS lysis buffer (2 \% SDS, $10 \%$ glycerol, $67.5 \mathrm{mM}$ Tris/ $\mathrm{Cl}^{-} \mathrm{pH}$ 6.8) and protease inhibitor cocktail. The samples were then heated to $95{ }^{\circ} \mathrm{C}$ while shaking (600 rpm for $20 \mathrm{~min}$ ) following which the supernatant was collected and stored at $-80{ }^{\circ} \mathrm{C}$ until further use. Protein concentration was measured using micro BCA (bicinchoninic acid) assay (Pierce, Rockford, IL). Total protein $(20 \mu \mathrm{g})$ was resolved using SDS-PAGE, transferred to nitrocellulose membranes and blocked in TBS-T/5\% milk for $1 \mathrm{~h}$. Membranes were incubated in primary antibody, calnexin (1:1000, rabbit polyclonal), calreticulin (1:1000,

Table 2 Details of the disease stage, chemotherapy treatment and disease outcome of the 11 rectal cancer patients of our validation cohort

\begin{tabular}{lllll}
\hline Patient & Stage & Neo-adjuvant therpay & RCPATH & Outcome \\
\hline 1 & III & $50.4 / 28+5 F U$ & A & Good \\
2 & III & $54.0 / 30+5 F U$ & A & Good \\
3 & III & $50.4 / 28+5 F U$ & A & Good \\
4 & II & $50.4 / 28+5 F U$ & A & Good \\
5 & III & $50.4 / 28+5 F U$ & B & Poor \\
6 & III & $50.4 / 28+5 F U$ & B & Poor \\
7 & III & $50.4 / 28+5 F U$ & B & Poor \\
8 & III & $50.4 / 28+5 F U$ & B & Poor \\
9 & III & $50.4 / 28+5 F U$ & C & Poor \\
10 & III & $50.4 / 28+5 F U$ & C & Poor \\
11 & II & $50.4 / 28+5 F U$ & C & Poor \\
\hline
\end{tabular}

5FU 5-fluorouracil; 50.4/28, total amount of 50.4 Gy radiotherapy in 28 fractions mouse monoclonal) and KDEL (1:500, mouse monoclonal) (Enzo Life Sciences, Exeter, UK) overnight at $4{ }^{\circ} \mathrm{C}$, washed in TBS-T and incubated in the appropriate horseradish peroxidase secondary antibody at room temperature for $1 \mathrm{~h}$. The KDEL antibody used binds the amino acid sequence Lys-Asp-Glu-Leu (KDEL) present at the carboxy-terminus of GRP78 and GRP94 thus this antibody detects both GRP78 and GRP94. Detection of protein bands was carried out using chemiluminescence (EMD Millipore, Billerica, MA, USA) on a LAS-3000 Imager (FUJIFILM UK Ltd. Systems, Bedford UK). Densitometry on each band was carried out using ImageJ software and normalized to Actin (1:5000 mouse monoclonal, Sigma Aldrich, Dublin, Ireland) loading control. Actin normalized intensities were then used to determine tumor to matched normal ratios for each patient.

\section{Calnexin gene silencing in HCT116 cells}

HCT116 colon cancer cells were transfected with calnexin siRNA and control scramble siRNA (Santa Cruz Biotechnology, Dallas, TX, USA). The cells were seeded on 6 -well tissue culture plates $\left(2 \times 10^{5}\right.$ cells/well $)$ and transfected after $24 \mathrm{~h}$. After a further $24 \mathrm{~h}$, cells were harvested to confirm knockdown of calnexin expression by western blot analysis.

\section{Flow cytometry}

Following transfection of HCT116 cells with calnexin or control scramble siRNA for $24 \mathrm{~h}$ and subsequent treatment with 5FU or vehicle for $48 \mathrm{~h}$, cells were harvested and the induction of cell death was assessed by Annexin $\mathrm{V}$ and PI staining using flow cytometry. Cells were incubated at room temperature in binding buffer $(10 \mathrm{mM}$ HEPES, $135 \mathrm{mM} \mathrm{NaCl}, 5 \mathrm{mM} \mathrm{CaCl}{ }_{2}$ ) which contained an Annexin V-FITC conjugate $(1 \mu \mathrm{l} / \mathrm{ml}$; BioVision, Mountain View, CA, USA) and propidium iodide (PI; $1 \mu \mathrm{l} / \mathrm{ml}$, BioVision) for $15 \mathrm{~min}$. Cells were counted in a Cyflow ML 16 flow cytometer (Partec, Münster, Germany). Excitation of Annexin V-FITC was done with a $488 \mathrm{~nm}$ laser and fluorescence emission was collected in the FL1 channel through a $520 \mathrm{~nm}$ band pass filter. PI was excited with a $488 \mathrm{~nm}$ laser and fluorescence emission was collected in the FL3 channel through a $620 \mathrm{~nm}$ long pass filter. $1 \times 10^{4}$ gated cells were acquired for each sample and analyzed using the Flowmax software (Partec).

\section{Clonogenic survival assay}

HCT116 cells were transfected with calnexin or control scramble siRNA for $24 \mathrm{~h}$ and then plated for a clonogenic survival assay. Transfected cells were seeded on a 6-well plate $\left(1 \times 10^{3}\right.$ cells/well $)$ in medium $(3 \mathrm{ml})$ and incubated at $37^{\circ} \mathrm{C}$ for 5 days. After 5 days, the medium was replaced with fresh medium and cells were incubated for a further 
9 days at which time medium was removed and clonogenic reagent ( $2 \mathrm{ml}$; $50 \%$ ethanol, $0.25 \% 1$, 9 dimethlyene blue) was added to each well and incubated at room temperature for $45 \mathrm{~min}$. The cells were then washed with PBS and the number of colonies was counted.

\section{Immunohistochemistry}

Tissue sections were cut at $3 \mu \mathrm{m}$ on to Leica Microsystems Plus slides and were baked over night at $37^{\circ} \mathrm{C}$ prior to immunostaining. All staining was performed on a Leica Bond III automated immunostainer from Leica Biosystems, Newcastle, UK. Sections were loaded onto the system and the relevant programme was selected. The Bond-III system dewaxed slides and then carried out a 20 min pre-treatment with BOND Epitope Retrieval Solution I (Cat. no. AR9961). Antibodies were chosen based on their suitability for use on FFPE tissue and optimised. Primary antibodies were diluted in Bond Primary Antibody Diluent (Cat. no. AR9352)-1:400 diluted rabbit polyclonal anti-Calnexin (ADI-SPA-860, Enzo Life Sciences, Exeter, UK) and were added to the sections for $20 \mathrm{~min}$. Detection and visualisation of stained cells was achieved using the Bond Polymer Refine Detection Kit (Cat. no. DS9800), using diaminobenzidine tetrachloride $(\mathrm{DAB})$ as the chromagen. Tissues were counterstained with haematoxylin and were cover slipped. Negative controls for rabbit antibody (Negative Control Rabbit Immunoglobulin fraction of serum from non-immunized rabbits, IS600, Dako) were included and no staining was observed in these controls.

\section{Statistical analysis}

All results from the patient samples and cell line work were analysed using GraphPad InStat software. The expression of individual proteins in tumour and matched normal samples was examined with two-sided Wilcoxon signed-rank test for related variables. The expression of the tumour to normal ratios was calculated using MannWhitney $U$ test for independent samples. In vitro data were analysed by basic $t$ tests and one-way ANOVA with Student-Newman-Keuls post hoc test where appropriate. Results were considered significant when the p-value was $<0.05$.

\section{Results}

\section{Expression of ER Stress proteins in stage II and stage III colorectal cancer patient samples}

The activation of the UPR in response to ER stress has the potential to allow cancer cells to survive adverse conditions that may otherwise lead to apoptosis. In order to examine the potential role of ER stress in colorectal cancer we determined the expression of the ER stress proteins calreticulin (Fig. 1b), calnexin (Fig. 1c), GRP78
(Fig. 1d) and GRP94 (Fig. 1e) in colorectal tumour and matched normal tissue from patients with Stage II $(\mathrm{n}=8)$ and Stage III $(\mathrm{n}=15)$ disease (Additional file 1: Table S1). Sample images of the Western blots of the ER stress proteins can be seen in Fig. 1a. A two-sided Wilcoxon signed rank test analysis of the expression of the ER stress proteins in the tumour samples and matched normal tissue established that there was no differential expression of the proteins between tumour and normal tissue (Fig. 1b, c, d, e). Furthermore, the levels of GRP78 and GRP94 were very low across the total patient cohort (Fig. 1d, e respectively). Additional file 2: Figure S1 provides an additional analysis with paired samples highlighted.

\section{Expression of ER stress proteins as a predictor of disease stage}

Since there was no differential expression of ER stress proteins between tumour and normal tissue in our CRC patient samples, we next assessed if the ratio of ER stress

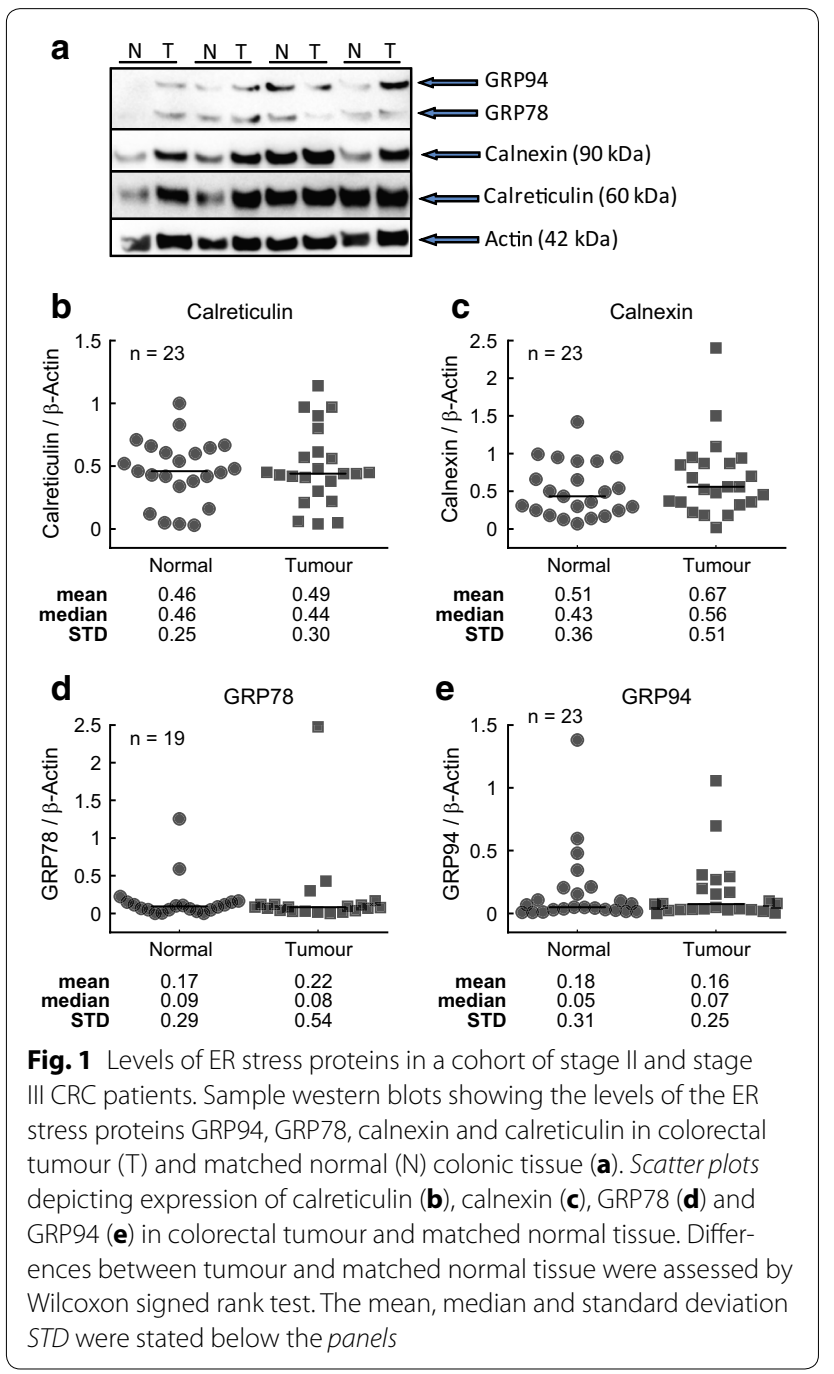


protein expression in tumour compared to normal tissue could be predictive of disease stage. We preferred to perform a tumour/normal ratio analysis to better control for potential differences in sample acquisition times and sample preparations among individually collected patient samples. Patient samples were subdivided by disease stage, stage II and stage III, and the ratio of calreticulin (Fig. 2a), calnexin (Fig. 2b), GRP78 (Fig. 2c) and GRP94 (Fig. 2d) in tumour versus normal tissue was evaluated. This analysis determined that there was no statistically significant difference between the ratios of ER stress protein expression in tumour versus normal tissue in samples from patients with Stage II CRC compared with samples from patients with Stage III CRC. Thus, the tumour/normal ratios of ER stress proteins are not predictive of disease stage. We likewise found no differences when comparing tumour and matched normal samples independently (Additional file 3: Figure S2).

\section{Expression of ER stress proteins as predictors of clinical outcome}

Since ER stress may alter tumour growth and chemosensitivity of tumour cells, it may also influence clinical outcomes and patient survival. Therefore, we next examined whether the expression of ER stress associated proteins in tumour tissue compared to normal

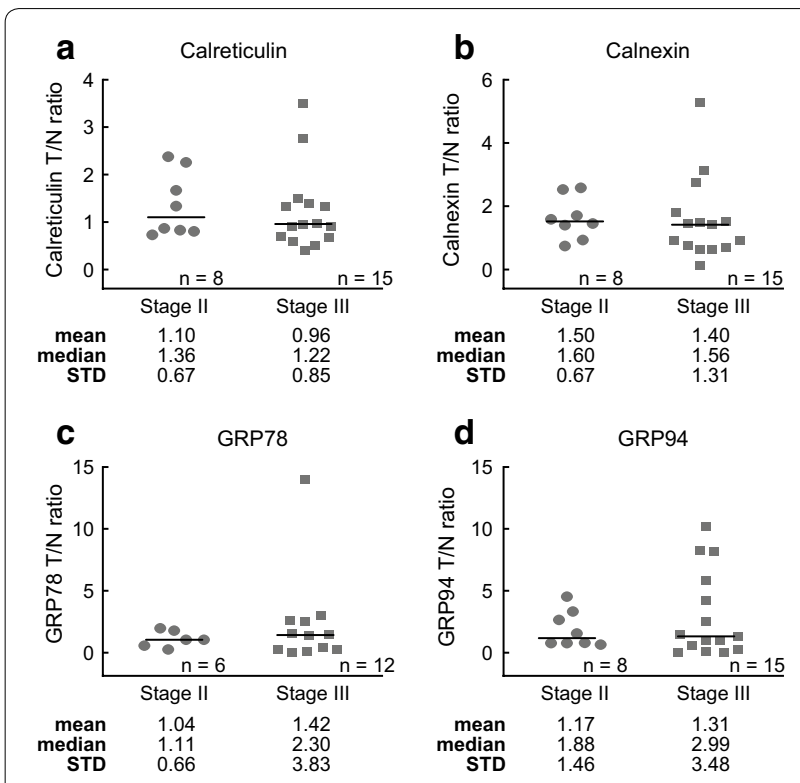

Fig. 2 Levels of ER stress proteins in CRC tissue do not correlate with disease stage. Scatter plots showing the tumour/normal ratio of the ER stress proteins calreticulin (a), calnexin (b), GRP78 (c) and GRP94 (d) in stage II versus stage III CRC patient tissue. Differences between tumour/normal ratios of the ER stress protein in stage II and stage III CRC were assessed by Mann-Whitney $U$ test. The mean, median and STD were stated below the panels tissue was associated with clinical outcome. Patient samples were divided based on the clinical outcome of the patients with good outcome being defined as no mortality or disease recurrence within the 4 year followup period while poor outcome was defined as disease recurrence or death from disease within that timeframe. The tumour/normal ratios of calnexin $(\mathrm{p}=0.0055$; Fig. 3b) were significantly increased in samples from patients who had poor clinical outcome. No significant differences in the tumour/normal ratios of calreticulin (Fig. 3a), GRP78 (Fig. 3c) and GRP94 (Fig. 3d) were detected. We likewise found elevated protein levels of calnexin in tumour ( $p=0.0030)$ but not matched normal tissue of patients with poor outcome when comparing tumour and matched normal samples independently (Additional file 4: Figure S3). In a separate analysis, we analysed only those patients who received 5FU based adjuvant chemotherapy. The tumour/normal ratios of calnexin ( $p=0.0093$; Additional file 5: Figure S4B) were significantly increased in samples from patients who received chemotherapy and had poor clinical outcome. No significant differences in the expression of calreticulin, GRP78 and GRP94 in tumour compared to matched

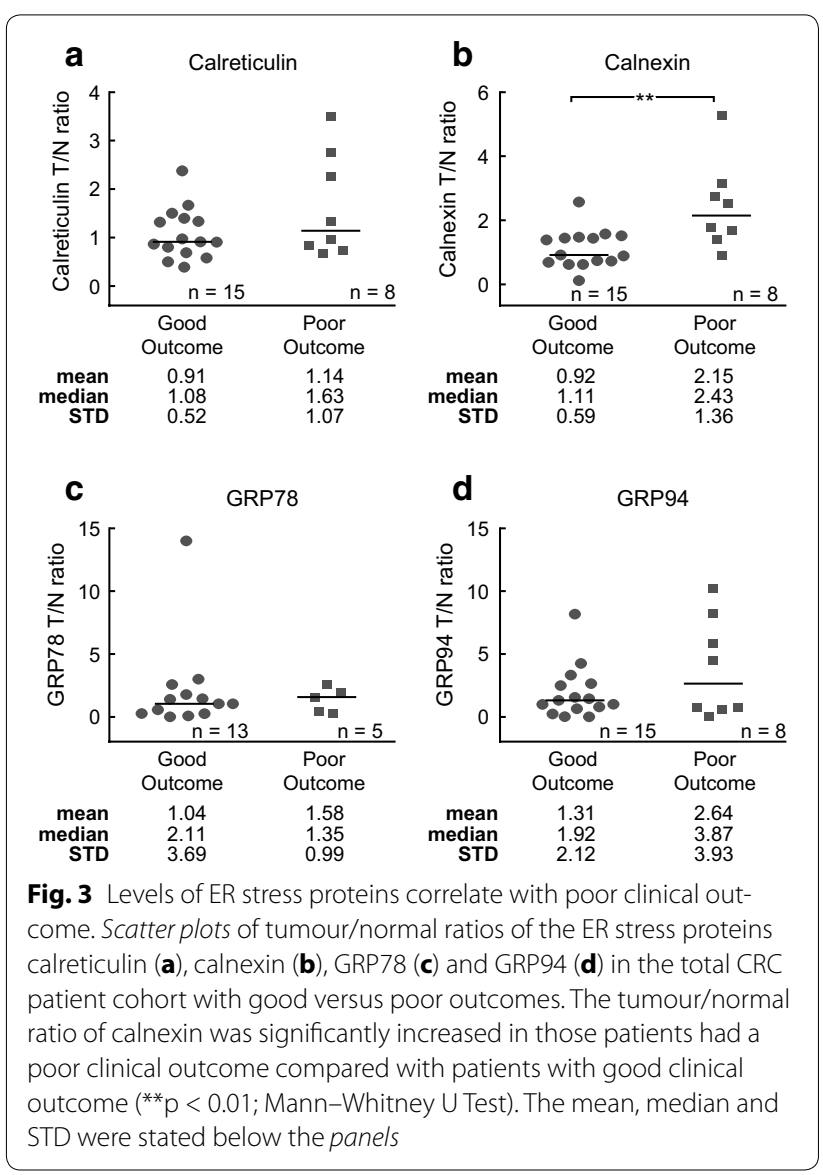


normal tissue were detected in samples from patients with good or poor outcome who received chemotherapy (Additional file 5: Figure S4A, C, D).

We also explored whether there were potential differences in the subcellular localization of calnexin in patients with good or poor outcome, and performed an immunohistochemical analysis of in good and in poor outcome cases. However, we observed little variation in the subcellular localization of calnexin in good and poor responders, with cytoplasmic, punctuate calnexin staining in all tumor cells (Fig. 4).

\section{Calnexin is a prognostic biomarker in rectal cancer patients treated in the neoadjuvant setting}

To validate our findings, pre-treatment biopsy tumour tissue samples were obtained at colonoscopy or rigid sigmoidoscopy (Table 1) from 11 rectal cancer patients. Using quantitative Western blotting, the expression levels of calnexin in tumour biopsy tissue were determined in all 11 patients (Additional file 1: Table S2). After neoadjuvant chemoradiotherapy $(50.4 / 28+5 \mathrm{FU})$, patients' response to therapy was determined on surgical resected tissue. Patients were either classified as good outcome (RCPath A; complete tumour regression) or bas outcome [RCPath B (partial tumour regression) or RCPath C (no marked tumour regression)].

We found statistically significant differences of the Calnexin level between the RCPaths (Kruskal-Wallis test $\mathrm{p}=0.0295$ ). After adjustment for multi-comparison (Bonferroni correction, $\alpha=0.0125$ ), Calnexin levels were significant up-regulated in poor responders (RCPath $B$ or C), compared to good responders (RCPath A; MannWhitney $\mathrm{U}$ test $\mathrm{p}=0.0286$ ) (Fig. 5).
The effect of calnexin knockdown on clonogenic survival and the induction of cell death by 5FU in HCT116 cells To determine a role for calnexin in colorectal cancer cell survival and 5FU-induced cell death, we silenced calnexin gene expression in HCT116 cells using siRNAmediated gene silencing. We first ascertained a successful reduction of calnexin protein levels in HCT116 cells $24 \mathrm{~h}$ post calnexin siRNA transfection as determined by Western blot analysis (Fig. 6a). A clonogenic survival assay was utilised to assess the ability of HCT116 cells to survive and proliferate following calnexin gene silencing. Gene silencing of calnexin significantly decreased clonogenic survival ( $\mathrm{p}=0.0021$, Fig. $6 \mathrm{~b}$ ).

Since calnexin expression was significantly elevated in patients with poor outcome who received 5FU-based chemotherapy, we next examined the effect of calnexin knockdown on chemosensitivity in HCT116 cells. Following transfection with calnexin siRNA for $24 \mathrm{~h}$, HCT116 cells were treated with $5 \mathrm{FU}(20 \mu \mathrm{g} / \mathrm{ml})$ for $48 \mathrm{~h}$ and the induction of cell death was determined by Annexin V and PI staining using flow cytometry. The percentage of Annexin $\mathrm{V}^{+}$cells, indicating apoptosis, was significantly increased when HCT116 cells transfected with calnexin siRNA and treated with 5FU were compared with HCT116 cells transfected with control siRNA and treated with 5FU [Fig. 6c, "p $<0.05$ versus HCT116 cells transfected with control siRNA and treated with 5FU]. Treatment with 5FU significantly increased the percentage of $\mathrm{PI}^{+}$cells in both control and calnexin siRNA transfected HCT116 cells with no significant effect of calnexin knockdown observed [Fig. $6 \mathrm{~d}$, ${ }^{* * *} \mathrm{p}<0.01$ versus HCT116 cells transfected with control siRNA and treated with vehicle; ${ }^{\# \#} \mathrm{p}<0.001$ versus $\mathrm{HCT} 116$ cells transfected

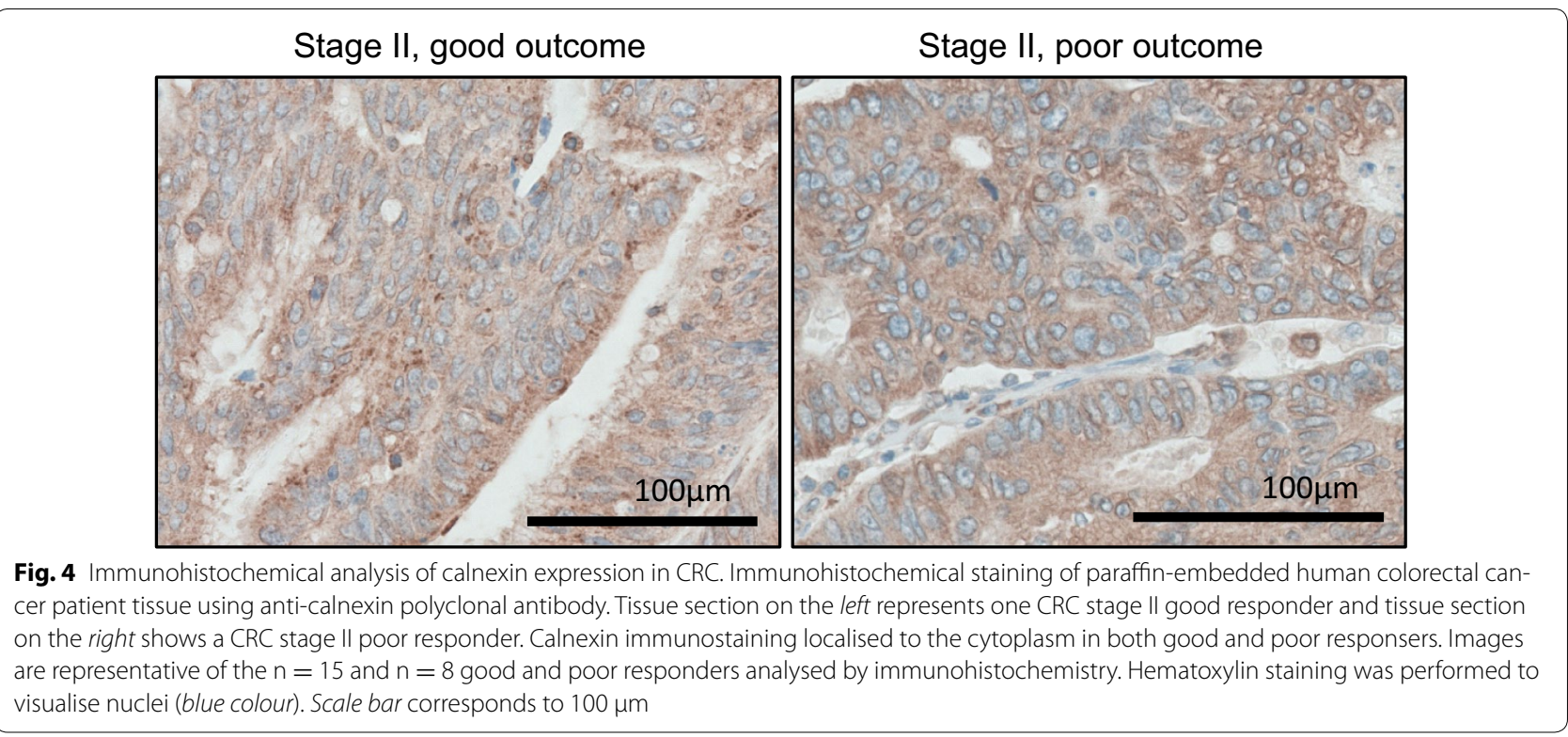




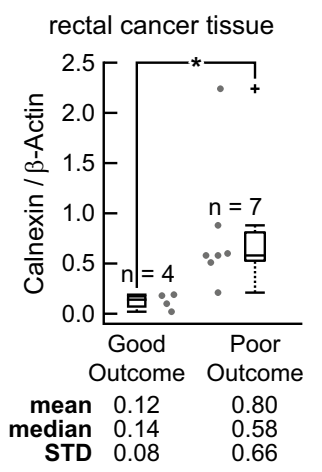

Fig. 5 Calnexin levels correlate with poor clinical outcome in rectal cancer patients treated in the neoadjuvant setting. The calnexin level was significantly increased in rectal cancer patients who received neo-adjuvant radiochemotherapy (50.4 Gy $+5 \mathrm{FU})$ and had a poor clinical outcome (RCPath B \& C) compared with patients who received neo-adjuvant radiochemotherapy with good clinical outcome (RCPath A; ${ }^{*} \mathrm{P}=0.0061$; Mann-Whitney U Test; Bonferroni corrected significance level $a=0.0125$ ) with calnexin siRNA and treated with vehicle]. While treatment of control siRNA transfected HCT116 cells with $5 \mathrm{FU}$ did not significantly increase the percentage of Annexin ${ }^{+} \mathrm{PI}^{+}$cells, knockdown of calnexin synergised with 5FU treatment to significantly enhance the percentage of HCT116 Annexin ${ }^{+} \mathrm{PI}^{+}$cells [Fig. 6e, ${ }^{* *} \mathrm{p}<0.01$ versus $\mathrm{HCT} 116$ cells transfected with control siRNA and treated with 5FU].

\section{Discussion}

The significant findings of this study identify the ER calcium binding chaperone calnexin as promising prognostic marker and therapeutic target in colorectal cancer. We have demonstrated that Calnexin levels correlated with clinical response in the total population of stage 2 and 3 CRC patients and in patients who received 5FU-based adjuvant chemotherapy. These findings were validated in rectal cancer patients treated with chemoradiation therapy in the neoadjuvant setting. Furthermore, we establish

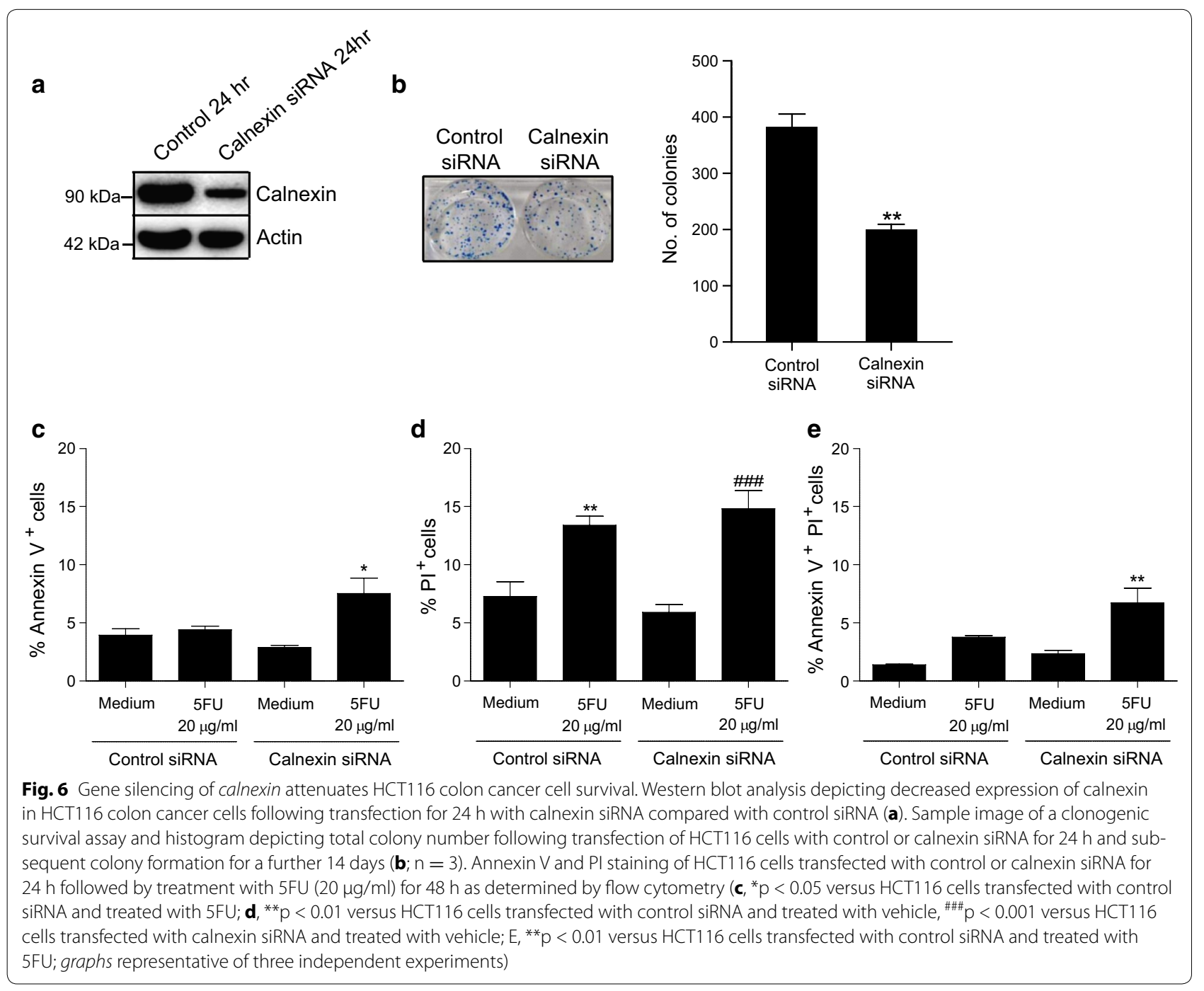


the importance of calnexin in colorectal cancer cell survival and responsiveness to 5FU-based chemotherapy.

The rapid proliferation of tumour cells requiring increased protein translation coupled with the inadequate blood supply has been suggested to lead to ER stress and activation of the UPR [8]. A tumour cell's ability to tolerate ER stress may account for its capacity to grow under adverse conditions and to resist current chemotherapeutic regimens. The ER resident chaperone GRP78, which plays a key role in sensing misfolded proteins and activating the UPR, has been shown to be up regulated in a variety of cancer cell lines and human specimens including breast, lung, liver and prostate cancer $[7,12]$. However, we demonstrate that the expression of GRP78 was low in both tumour and matched normal tissue in our colorectal cancer patient samples and the expression levels of GRP78 failed to correlate with disease stage or clinical outcome. In contrast to our findings, other studies have demonstrated that the level of GRP78 positively correlated with tumour progression in primary human breast [13], liver [14] and melanoma [15] tumour tissue. Interestingly, a study by Hardy et al. [16] showed that the localisation of GRP78 within colon cancer cells was crucial to their tumourigenicity with cells expressing GRP78 in the ER but not on the cell surface being highly proliferative and capable of inducing liver metastasis in a human metastatic colorectal carcinoma model in mice [16]. While we did not detect differences in the subcellular localisation of calnexin by immunohistochemistry among good and poor responders, it is possible that the localisation of GRP78 and not its overall expression level or tumour/normal ratio determines its contribution to disease stage or clinical outcome of the patient. This was not further analysed in this study as we were not able to identify a solely GRP78 specific antibody compatible with FFPE samples.

We also demonstrate that the glucose regulated ER resident molecular chaperone GRP94 is lowly expressed in our colorectal cancer tumour and matched normal samples. Previous studies indicate that overexpression of GRP94 associated with cellular transformation and tumourigenicity in cancer cell lines [17]. In contrast, GRP94 is known to elicit anti-tumour immune responses in mouse models $[18,19]$ through its ability to bind and present tumour specific antigens [20] and induce maturation of dendritic cells [21]. However, we found no evidence that the expression of GRP94 correlated with disease stage or clinical outcome in our cohort of CRC patients.

Calcium, the universal signaling molecule involved in processes such as protein modification, protein folding and cell survival and death signaling, is stored in the ER, where it is buffered by that calcium binding ER chaperones calreticulin and calnexin. Our data demonstrate that an increased tumour/normal ratio of calnexin, but not calreticulin, was significantly associated with poor clinical outcome in patients that received chemotherapy. Calreticulin has been implicated in affecting cell sensitivity to apoptosis, with calreticulin deficiency lowering ER luminal $\mathrm{Ca}^{2+}$ concentrations and protecting cells from apoptosis [22] while overexpression of calreticulin sensitizes cells to the induction of apoptosis [22, 23]. Expression of the calcium binding ER chaperone calnexin has been shown to be a good predictor of cell sensitivity to ER stress-mediated cell death [24] and to mediate resistance to apoptosis through its caspase-dependent cleavage [25]. In the present study, we significantly expand on the key role of calnexin in colon cancer cell survival by demonstrating that gene silencing of calnexin decreases clonogenic cell survival. Furthermore, we show that gene silencing of calnexin in combination with 5FU treatment significantly increases the percentage of cells undergoing both early (Annexin $\mathrm{V}^{+}$only cells) and late apoptosis (Annexin $\mathrm{V}^{+} \mathrm{PI}^{+}$cells) as well as inducing significant levels of necrosis ( $\mathrm{PI}^{-}$only cells and Annexin $\mathrm{V}^{+} \mathrm{PI}^{+}$cells). It has been previously demonstrated that cells defective in apoptosis undergo autophagy-induced necrotic-like cell death as a result of prolonged ER stress [26], which could explain the induction of necrosis observed in our study.

CRC patients with tumors exhibiting high microsatellite instability (MSI) were previously shown not to benefit from 5FU-based adjuvant chemotherapy [27]. Our finding might suggest that, targeting calnexin in combination with 5FU treatment might improve overall survival among patients with high MSI, since HCT-116 cells are known to feature clinical and cytological characteristics of CRC patients stratified for high MSI [28].

\section{Conclusions}

In summary, this study indicates that the calnexin protein levels may represent a new indicator of poor clinical outcome of stage II/III colorectal cancer patients who received 5FU-based chemotherapy. Furthermore, our in vitro studies confirmed the importance of calnexin for colorectal cancer cell growth and proliferation and also demonstrated that calnexin deficiency could enhance responsiveness to 5FU-based-chemotherapy. Together, these results suggest a possible role for the calcium binding chaperone calnexin as a prognostic biomarker in stage 2 and 3 colorectal cancer and as a potential therapeutic target, which requires further validation in clinical and pre-clinical studies. 


\section{Additional files}

Additional file 1: Table $\mathbf{S} 1$ and $\mathbf{S 2}$. Densitometric levels for ER stress proteins calreticulin, calnexin, GRP78 and GRP94 in CRC patients by quantitive western blotting.

Additional file 2: Figure S1. Box and scatter plots depicting expression of calreticulin (A), calnexin (B), GRP78 (C) and GRP94 (D) in colorectal tumour and matched normal tissue. Differences between tumour and matched normal tissue were assessed by Wilcoxon signed rank test. Paired samples with lower levels in tumour compared to matched normal tissue were mapped with a solid line. Paired samples with higher levels in tumour compared to matched normal tissue were mapped with a dotted line.

Additional file 3: Figure S2. Box plots showing tumour and matched normal levels of the ER stress proteins calreticulin (A), calnexin (B), GRP78 (C) and GRP94 (D) in stage II versus stage III CRC patient tissue. Differences between expression levels of the ER stress proteins in stage II and stage III CRC were assessed by Mann Whitney $U$ test.

Additional file 4: Figure S3. Box plots of tumour and matched normal levels of the ER stress proteins calreticulin (A), calnexin (B), GRP78 (C) and GRP94 (D) in the total CRC patient cohort in good versus poor outcome cases. The expression level of calnexin in tumour tissue was significantly increased in patients with poor clinical outcome compared with patients with good clinical outcome ${ }^{* *} p<0.01$; Mann Whitney U Test).

Additional file 5: Figure S4. Levels of calnexin correlate with poor clinical outcome in patients that received adjuvant chemotherapy. Scatter plots of tumour/normal ratios of the ER stress proteins calreticulin (A), calnexin (B), GRP78 (C) and GRP94 (D) in patients who received 5FU based adjuvant chemotherapy. The tumour/normal ratio of calnexin was significantly increased in those patients who received adjuvant chemotherapy and had a poor clinical outcome compared with patients who received adjuvant chemotherapy with good clinical outcome ${ }^{* *} p<0.01$; Mann Whitney U Test).

\section{Abbreviations}

5FU: 5-fluorouracil; BCA: bicinchoninic acid; CRC: colorectal cancer; DAB: diaminobenzidine tetrachloride; EDTA: ethylenediaminetetraacetic acid; ER: endoplasmic reticulum; Fol: fluorouracil; GRP78: 78 kDa glucose-regulated protein/binding immunoglobulin protein; GRP94: heat shock protein $90 \mathrm{kDa}$ beta member 1; Irin: irinotecan; h: hours; HEPES: 2-[4-(2-hydroxyethyl)piperazin-1-yl] ethanesulfonic acid; KDEL: amino acid sequence Lys-Asp-Glu-Leu; Leu: leucovorin; M: molar; min: minute; MSI: microsatellite instability; Oxal: oxaliplatin; p: $p$ value; PI: propidium iodide; RCPath: standard to describe tumour regression following neoadjuvant therapy published by the Royal College of Pathologists; rpm: revolutions per minute; SDS: sodium dodecyl sulfate; siRNA: small interfering RNA; Suppl.: supplementary; UPR: unfolded protein response.

\section{Authors' contributions}

$\mathrm{DR}, \mathrm{ACM}, \mathrm{SC}$ and JHMP designed the study, interpreted the data and wrote the paper. DR, ACM, JF, SC and SH carried out the experiments. DR and AUL performed the statistically analysis. NMCC, OB, EWK, DAMCN participated in studies coordination, and provided administrative, technical, or material support. CGC and JHMP supervised the project. All authors reviewed and revised the final manuscript. All authors read and approved the final manuscript.

\section{Author details}

1 Department of Physiology and Medical Physics, Centre for Systems Medicine, Royal College of Surgeons in Ireland, 123 St Stephen's Green, Dublin 2, Ireland. ${ }^{2}$ Department of Colorectal Surgery, Beaumont Hospital, Dublin 9, Ireland.

${ }^{3}$ Department of Pathology, Beaumont Hospital and Royal College of Surgeons in Ireland, Dublin 9, Ireland.

\section{Acknowledgements}

We gratefully acknowledge the help of Ms. Joan Kehoe in Beaumont Hospital.

\section{Competing interests}

The authors declare that they have no competing interests.

\section{Funding}

This research was supported by grants from the Health Research Board (TRA/2007/26), Science Foundation Ireland (13/IA/1881) and the European Union (FP7 APO-DECIDE Contract 306021) to JHMP.

Received: 24 August 2015 Accepted: 20 June 2016

Published online: 01 July 2016

\section{References}

1. Yang SY, Sales KM, Fuller B, Seifalian AM, Winslet MC. Apoptosis and colorectal cancer: implications for therapy. Trends Mol Med. 2009;15(5):22533. doi:10.1016/j.molmed.2009.03.003.

2. Figueredo A, Coombes ME, Mukherjee S. Adjuvant therapy for completely resected stage II colon cancer. Cochrane Database Syst Rev. 2008;3:CD005390.

3. Ragnhammar P, Hafstrom L, Nygren P, Glimelius B. A systematic overview of chemotherapy effects in colorectal cancer. Acta Oncol. 2001;40(2-3):282-308.

4. Gray R, Barnwell J, McConkey C, Hills RK, Williams NS, Kerr DJ. Adjuvant chemotherapy versus observation in patients with colorectal cancer: a randomised study. Lancet. 2007;370(9604):2020-9. doi:10.1016/ S0140-6736(07)61866-2.

5. de Gramont A, Figer A, Seymour M, Homerin M, Hmissi A, Cassidy J, et al. Leucovorin and fluorouracil with or without oxaliplatin as first-line treatment in advanced colorectal cancer. J Clin Oncol. 2000;18(16):2938-47.

6. Wang G, Yang ZQ, Zhang K. Endoplasmic reticulum stress response in cancer: molecular mechanism and therapeutic potential. Am J Transl Res. 2010;2(1):65-74.

7. Ni M, Lee AS. ER chaperones in mammalian development and human diseases. FEBS Lett. 2007;581(19):3641-51. doi:10.1016/j. febslet.2007.04.045.

8. Feldman DE, Chauhan V, Koong AC. The unfolded protein response: a novel component of the hypoxic stress response in tumors. Mol Cancer Res. 2005;3(11):597-605. doi:10.1158/1541-7786.MCR-05-0221.

9. Corazzari M, Rapino F, Ciccosanti F, Giglio P, Antonioli M, Conti B, et al. Oncogenic BRAF induces chronic ER stress condition resulting in increased basal autophagy and apoptotic resistance of cutaneous melanoma. Cell Death Differ. 2015;22(6):946-58. doi:10.1038/cdd.2014.183.

10. Schonthal AH. Endoplasmic reticulum stress and autophagy as targets for cancer therapy. Cancer Lett. 2009;275(2):163-9. doi:10.1016/j. canlet.2008.07.005.

11. Li X, Zhang K, Li Z. Unfolded protein response in cancer: the physician's perspective. J Hematol Oncol. 2011;4:8. doi:10.1186/1756-8722-4-8.

12. Jamora C, Dennert $G$, Lee AS. Inhibition of tumor progression by suppression of stress protein GRP78/BiP induction in fibrosarcoma B/C10ME. Proc Natl Acad Sci USA. 1996;93(15):7690-4.

13. Fernandez PM, Tabbara SO, Jacobs LK, Manning FC, Tsangaris TN, Schwartz AM, et al. Overexpression of the glucose-regulated stress gene GRP78 in malignant but not benign human breast lesions. Breast Cancer Res Treat. 2000;59(1):15-26.

14. Shuda M, Kondoh N, Imazeki N, Tanaka K, Okada T, Mori K, et al. Activation of the ATF6, XBP1 and grp78 genes in human hepatocellular carcinoma: a possible involvement of the ER stress pathway in hepatocarcinogenesis. J Hepatol. 2003;38(5):605-14.

15. Zhuang L, Scolyer RA, Lee CS, McCarthy SW, Cooper WA, Zhang XD, et al. Expression of glucose-regulated stress protein GRP78 is related to progression of melanoma. Histopathology. 2009;54(4):462-70. doi:10.1111/j.1365-2559.2009.03242.x.

16. Hardy B, Raiter A, Yakimov M, Vilkin A, Niv Y. Colon cancer cells expressing cell surface GRP78 as a marker for reduced tumorigenicity. Cell Oncol (Dordr). 2012;35(5):345-54. doi:10.1007/s13402-012-0094-4.

17. Fu Y, Lee AS. Glucose regulated proteins in cancer progression, drug resistance and immunotherapy. Cancer Biol Ther. 2006;5(7):741-4. 
18. Tamura Y, Peng P, Liu K, Daou M, Srivastava PK. Immunotherapy of tumors with autologous tumor-derived heat shock protein preparations. Science. 1997;278(5335):117-20

19. Blachere NE, Li Z, Chandawarkar RY, Suto R, Jaikaria NS, Basu S, et al. Heat shock protein-peptide complexes, reconstituted in vitro, elicit peptidespecific cytotoxic T lymphocyte response and tumor immunity. J Exp Med. 1997;186(8):1315-22.

20. Schild H, Rammensee HG. gp96-the immune system's Swiss army knife. Nat Immunol. 2000;1(2):100-1.

21. Singh-Jasuja H, Scherer HU, Hilf N, Arnold-Schild D, Rammensee HG, Toes RE, et al. The heat shock protein gp96 induces maturation of dendritic cells and down-regulation of its receptor. Eur J Immunol. 2000;30(8):2211-5.

22. Nakamura K, Bossy-Wetzel E, Burns K, Fadel MP, Lozyk M, Goping IS, et al. Changes in endoplasmic reticulum luminal environment affect cell sensitivity to apoptosis. J Cell Biol. 2000;150(4):731-40.

23. Arnaudeau S, Frieden M, Nakamura K, Castelbou C, Michalak M, Demaurex N. Calreticulin differentially modulates calcium uptake and release in the endoplasmic reticulum and mitochondria. J Biol Chem. 2002;277(48):46696-705. doi:10.1074/jbc.M202395200.
24. Delom F, Emadali A, Cocolakis E, Lebrun JJ, Nantel A, Chevet E. Calnexindependent regulation of tunicamycin-induced apoptosis in breast carcinoma MCF-7 cells. Cell Death Differ. 2007;14(3):586-96. doi:10.1038/ sj.cdd.4402012.

25. Takizawa T, Tatematsu C, Watanabe K, Kato K, Nakanishi Y. Cleavage of calnexin caused by apoptotic stimuli: implication for the regulation of apoptosis. J Biochem. 2004;136(3):399-405. doi:10.1093/jb/mvh133.

26. Ullman E, Fan Y, Stawowczyk M, Chen HM, Yue Z, Zong WX. Autophagy promotes necrosis in apoptosis-deficient cells in response to ER stress. Cell Death Differ. 2008;15(2):422-5. doi:10.1038/sj.cdd.4402234.

27. Ribic CM, Sargent DJ, Moore MJ, Thibodeau SN, French AJ, Goldberg RM, et al. Tumor microsatellite-instability status as a predictor of benefit from fluorouracil-based adjuvant chemotherapy for colon cancer. New Engl J Med. 2003;349(3):247-57. doi:10.1056/NEJMoa022289.

28. Parsons R, Li GM, Longley MJ, Fang WH, Papadopoulos N, Jen J, et al. Hypermutability and mismatch repair deficiency in RER + tumor cells. Cell. 1993;75(6):1227-36.

\section{Submit your next manuscript to BioMed Central and we will help you at every step:}

- We accept pre-submission inquiries

- Our selector tool helps you to find the most relevant journal

- We provide round the clock customer support

- Convenient online submission

- Thorough peer review

- Inclusion in PubMed and all major indexing services

- Maximum visibility for your research

Submit your manuscript at www.biomedcentral com/submit 